COMMUNICATIONS IN

ANALYSIS AND GEOMETRY

Volume 4, Number 3, 435-457, 1996

\title{
Conformal flows by curvature on compact manifolds
}

\author{
LiI-PERNG LIOU
}

\section{Introduction}

Let $\left(M^{n}, g\right), n \geq 2$, be a connected compact $n$-dimensional manifold. We will consider the flow by the equation

$$
\frac{\partial \bar{g}}{\partial t}=F(\bar{R}) \bar{g}, \quad \bar{g}(0)=e^{2 f_{0}} g,
$$

where $\bar{g}$ is the metric, $\bar{R}$ is the scalar curvature, and $f_{0}$ is an arbitrary smooth function on $M$. We shall assume that $F: \mathbb{R} \rightarrow \mathbb{R}$ is a smooth function with $F^{\prime}<0$. The assumption that $F^{\prime}<0$ is to make (1.1) a parabolic equation.

Our work is motivated by the study of the following equation, which we refer to as Hamilton's Ricci flow when $n=2$ and Yamabe flow when $n \geq 3$ respectively:

$$
\frac{\partial \bar{g}}{\partial t}=(r-\bar{R}) \bar{g}
$$

where $r$ is the average of $\bar{R}$. Hamilton proved

Theorem 1.1. (Hamilton [5]). Let $(M, \bar{g})$ be a compact oriented Riemannian surface.

(1) If $M$ is not diffeomorphic to the 2-sphere $S^{2}$, then any metric $\bar{g}$ converges to a constant curvature metric under the Hamilton-Ricci flow.

(2) If $M$ is diffeomorphic to $S^{2}$, then any metric $\bar{g}$ with positive Gaussian curvature on $S^{2}$ converges to a curvature of constant metric under the Hamilton-Ricci flow.

Later on, Chow [2] removed the assumption in Hamilton's theorem that a metric on $S^{2}$ has positive scalar curvature.

Recently, a new proof to the Hamilton and Chow's results was given by Bartz, Struwe and Ye [1]. They used the Aleksandrov reflection method to prove a gradient estimate, which then easily yields uniform smooth estimates 
of the solution by linear theory and bootstrapping. Ye [11] also used a similar method to study the Yamabe flow on a compact manifold with dimension $n \geq 3$. The most important result in that paper is

Theorem 1.2. (Ye [11])Assume that $(M, g)$ is locally conformally flat with positive scalar curvature. Then for any smooth function $f_{0}$, the Yamabe flow has a unique smooth solution on the time interval $[0, \infty)$. Moreover, the solution metric $\bar{g}$ converges smoothly to a unique limit metric of constant scalar curvature as $t \rightarrow \infty$.

The purpose of this paper is to study more nonlinear analogues of the Yamabe flow on compact manifolds.

We write $\bar{g}=e^{2 f} g$ and let $R$ be the scalar curvature of $(M, g)$, define $G(s)=\frac{1}{2} F(2(n-1) s)$, then (1.1) becomes

$$
\left\{\begin{array}{l}
f_{t}=G\left(e^{-2 f}\left(\frac{R}{2(n-1)}-\Delta f-\frac{n-2}{2}|\nabla f|^{2}\right)\right) \\
f(0, x)=f_{0}
\end{array}\right.
$$

since

$$
\bar{R}=2(n-1) e^{-2 f}\left(\frac{R}{2(n-1)}-\Delta f-\frac{n-2}{2}|\nabla f|^{2}\right){ }^{1} .
$$

It is well-known that there is a unique solution to (1.2) on $M \times\left[0, T_{0}\right)$ for some short time $T_{0}$. In section 2 , we shall prove that the solution exists as long as $\|f(\cdot, t)\|_{C^{1}(M)}$ is bounded.

In section 3 , we prove that the scalar curvature $\bar{R}$ is exponentially decaying if we assume that $F>0$, which we refer to as the expanding flow. We also obtain a uniform gradient estimate for $f$ in section 3. In section 4, we prove the crucial Hessian estimate for the expanding flow. In section 5 and 6 we prove that $|\nabla \Delta f|$ and $|\Delta \Delta f|$ are uniformly bounded under the expanding flow. In section 7 , we use a bootstrapping argument to show that all the higher derivatives are uniformly bounded and prove our main result for the expanding flow. Our main result is the following

Theorem 1.3. (Expanding flow). Assume that $(M, g)$ is a connected compact manifold and $F>0$. Then the solution to (1.1) exists for all time and the rescaled metric $\widetilde{g} \equiv e^{-2 G(0) t} \bar{g}$ converges in $C^{\infty}$ norm to a smooth metric $\tilde{g}_{\infty}$ on $M$.

\footnotetext{
${ }^{1}$ Throughout this paper we will use $\Delta$ and $\nabla$ as the Laplacian and the covariant derivative with respect to the background metric $g$ on $M$ respectively.
} 
Like Hamilton interprets the Ricci flow as the Heat flow for the Ricci curvature, we can interpret the equation (1.1) as a nonlinear Heat flow for the scalar curvature function. Thus we believe that the metric $\tilde{g}_{\infty}$ should have some interesting geometric properties. It seems to be natural to conjecture that the scalar curvature of $\widetilde{g}_{\infty}$ is a constant.

\section{A general long time existence theorem.}

In studying equation (1.2), one of the main difficulties is to prove the long time existence. As mentioned in the introduction, in this section we shall prove that the solution $f$ to (1.2) exists until $\|f(\cdot, t)\|_{C^{1}(M)}$ blows up. More precisely, we shall prove the following

Theorem 2.1. Let $(M, g)$ be a connected compact manifold. If we denote $\left[0, T_{\infty}\right)$ the maximal time interval on which the solution $f$ to (1.2) exists, then either $T_{\infty}=\infty$ or $\sup _{\left[0, T_{\infty}\right)}\|f(\cdot, t)\|_{C^{1}(M)}=\infty$.

The idea of the proof of Theorem 2.1 is similar to the idea in [4]. We first need to compute a few evolution equations.

Lemma 2.2. The evolution equation for $\bar{R}$ is

$$
\partial_{t} \bar{R}=(1-n) e^{-2 f}[\Delta F(\bar{R})+(n-2) \nabla F(\bar{R}) \nabla f]-F(\bar{R}) \bar{R} .
$$

Proof. We differentiate (1.3) with respect to $t$ and use the equation $f_{t}=$ $\frac{1}{2} F(\bar{R})$, we have

$$
\begin{aligned}
& \partial_{t} \bar{R}=\left(-2 f_{t}\right) \bar{R}+2(n-1) e^{-2 f}\left(-\Delta f_{t}-(n-2) \nabla f_{t} \nabla f\right) \\
& =(1-n) e^{-2 f}[\Delta F(\bar{R})+(n-2) \nabla F(\bar{R}) \nabla f]-F(\bar{R}) \bar{R} .
\end{aligned}
$$

Let $F(\bar{R})=W$. From (2.1), it's easy to see that $W$ satisfies

$$
\begin{aligned}
& \partial_{t} W=(1-n) F^{\prime} \circ F^{-1}(W) e^{-2 f}[\Delta W+(n-2) \nabla W \nabla f] \\
& -F^{\prime} \circ F^{-1}(W) W \bar{R} .
\end{aligned}
$$

By straightforward computation it's also easy to see 
Lemma 2.3. The evolution equation for $|\nabla f|^{2}$ is

$$
\begin{aligned}
& \partial_{t}|\nabla f|^{2}=-G^{\prime} e^{-2 f}\left[\Delta|\nabla f|^{2}-2|\nabla \nabla f|^{2}-2 R_{i l} \nabla_{i} f \nabla_{l} f\right. \\
& \left.+4|\nabla f|^{2}\left(\frac{R}{2(n-1)}-\Delta f-\frac{(n-2)}{2}|\nabla f|^{2}\right)-\frac{\nabla f \nabla R}{(n-1)}+(n-2) \nabla f \nabla|\nabla f|^{2}\right],
\end{aligned}
$$

where $G^{\prime}=G^{\prime}\left(\frac{\bar{R}}{2(n-1)}\right)$ and $R_{i l}$ is the Ricci tensor on $M$ with respect to $g$.

The good term $2 G^{\prime} e^{-2 f}|\nabla \nabla f|^{2}$ in the evolution of $|\nabla f|^{2}$ may be used to estimate $W$ as follows. Let $\alpha$ be a positive constant to be chosen later, we consider the quantity $\phi=\left(|\nabla f|^{2}+\alpha\right) W$ and compute its evolution equation

$$
\begin{aligned}
& \partial_{t} \phi=(1-n) F^{\prime} e^{-2 f}\left[\Delta \phi-2 \nabla\left(|\nabla f|^{2}+\alpha\right) \nabla W-2|\nabla \nabla f|^{2} W\right. \\
& -2 R_{i l} \nabla_{i} f \nabla_{l} f W+4|\nabla f|^{2} W\left(\frac{R}{2(n-1)}-\Delta f-\frac{(n-2)}{2}|\nabla f|^{2}\right)-\frac{\nabla f \nabla R}{(n-1)} W \\
& \left.+(n-2) \nabla f \nabla|\nabla f|^{2} W+(n-2) \nabla W \nabla f\left(|\nabla f|^{2}+\alpha\right)\right]-F^{\prime} W \bar{R}\left(|\nabla f|^{2}+\alpha\right) .
\end{aligned}
$$

Here $F^{\prime}=F^{\prime}(\bar{R})$. We can write

$$
-2 \nabla\left(|\nabla f|^{2}+\alpha\right) \nabla W=\frac{-2 \nabla\left(|\nabla f|^{2}+\alpha\right) \nabla \phi}{|\nabla f|^{2}+\alpha}+\frac{\left.\left.2|\nabla| \nabla f\right|^{2}\right|^{2} W}{|\nabla f|^{2}+\alpha}
$$

and

$$
(n-2) \nabla W \nabla f\left(|\nabla f|^{2}+\alpha\right)+(n-2) W \nabla|\nabla f|^{2} \nabla f=(n-2) \nabla \phi \nabla f .
$$

We shall prove Theorem 2.1 by contradiction. Assume that $T_{\infty}<\infty$ and $\sup _{\left[0, T_{\infty}\right)}\|f(\cdot, t)\|_{C^{1}(M)}<\infty$. From (1.3) we have

$$
\Delta f=\frac{(2-n)}{2}|\nabla f|^{2}+\frac{R}{2(n-1)}-\frac{1}{2(n-1)} e^{2 f} \bar{R}
$$

Therefore $|\Delta f| \leq C_{1}+C_{2}|\bar{R}|$ and $|\Delta f|^{2} \geq C_{3} \bar{R}^{2}-C_{4}$. Throughout this proof, all the constants $C_{i}$ may depend on $T_{\infty}$ and $\sup _{\left[0, T_{\infty}\right)}\|f(\cdot, t)\|_{C^{1}(M)}$. Using that $\sup _{\left[0, T_{\infty}\right)}\|f(\cdot, t)\|_{C^{1}(M)}$ is bounded and the inequality $|\nabla \nabla f|^{2} \geq \frac{1}{n}|\Delta f|^{2}$, if we 


$$
\begin{aligned}
& \text { choose } \alpha=9 \sup _{M \times\left[0, T_{\infty}\right)}|\nabla f|^{2} \text { then } \\
& \qquad \begin{array}{l}
\frac{\left.\left.2|\nabla| \nabla f\right|^{2}\right|^{2}}{|\nabla f|^{2}+\alpha}-2|\nabla \nabla f|^{2}+4|\nabla f|^{2}\left(\frac{R}{2(n-1)}-\Delta f-\frac{(n-2)}{2}|\nabla f|^{2}\right)-\frac{\nabla f \nabla R}{(n-1)} \\
\quad-2 R_{i l} \nabla_{i} f \nabla_{l} f-\frac{1}{(1-n)} e^{2 f} \bar{R}\left(|\nabla f|^{2}+\alpha\right) \\
\leq\left(\frac{8|\nabla f|^{2}|\nabla \nabla f|^{2}}{|\nabla f|^{2}+\alpha}-|\nabla \nabla f|^{2}\right)-\frac{1}{n}\left(C_{3} \bar{R}^{2}-C_{4}\right)+C_{5}|\bar{R}|+C_{6} \\
\leq-C_{7} \bar{R}^{2}+C_{8} .
\end{array}
\end{aligned}
$$

Let $\phi_{\max }=\max _{x \in M} \phi(x, t)$ then by maximum principle and that $F^{\prime}<0$, we have

$$
\frac{d}{d t} \phi_{\max } \leq 0 \text { if } \phi_{\max } \geq \max \left\{0, \sup _{M \times\left[0, T_{\infty}\right)}\left(|\nabla f|^{2}+\alpha\right) F\left(-\sqrt{\frac{C_{8}}{C_{7}}}\right)\right\},
$$

which proves that $\phi_{\max } \leq C_{9}$. We remark that $\phi_{\max }$ is only a Lipschitz function and inequality (2.4) is interpreted as in section 3 of [7]. A lower bound on $\phi$ can be proved analogously, hence we have shown that $F(\bar{R})$ is bounded. The next step is to show that $\bar{R}$ is bounded. By the evolution of $\bar{R}$

$$
\partial_{t} \bar{R}=(1-n) e^{-2 f}\left[F^{\prime} \Delta \bar{R}+F^{\prime \prime}|\nabla \bar{R}|^{2}+(n-2) F^{\prime} \nabla \bar{R} \nabla f\right]-F \bar{R}
$$

using the estimate on $F(\bar{R})$ and applying maximum principle, it is easy to prove that $\bar{R}$ is bounded on $\left[0, T_{\infty}\right)$.

To get a contradiction, we still need to have all the higher derivatives bounded. We again consider the equation for $W$

$$
\begin{aligned}
& \partial_{t} W=(1-n) F^{\prime} \circ F^{-1}(W) e^{-2 f}[\Delta W+(n-2) \nabla W \nabla f] \\
& -F^{\prime} \circ F^{-1}(W) W F^{-1}(W) .
\end{aligned}
$$

Since both $W$ and $\bar{R}$ are bounded, $F^{\prime} \circ F^{-1}(W)$ is also bounded, hence we may apply Theorem 4.2 in [10] to conclude that $\|W\|_{C^{\alpha}\left(M \times\left[0, T_{\infty}\right)\right)} \leq C$, which implies that $\|\bar{R}\|_{C^{\alpha}\left(M \times\left[0, T_{\infty}\right)\right)} \leq C$ and $\left\|F^{\prime} \circ F^{-1}(W)\right\|_{C^{\alpha}\left(M \times\left[0, T_{\infty}\right)\right)} \leq$ $C$. To get $\|W\|_{C^{2, \alpha}\left(M \times\left[0, T_{\infty}\right)\right)}$

bounded, we need $\nabla f$ being bounded in $C^{\alpha}$ norm. For this purpose, we first 
observe that $\sup _{\left[0, T_{\infty}\right)}\|f(\cdot, t)\|_{C^{2, \alpha}(M)} \leq C$ from (2.3) and linear elliptic theory. We then look at the evolution of $\nabla_{i} f$

$$
\begin{aligned}
& \partial_{t} \nabla_{i} f=-G^{\prime} e^{-2 f}\left[\Delta \nabla_{i} f-R_{i l} \nabla_{l} f+2 \nabla_{i} f\left(\frac{R}{2(n-1)}-\Delta f-\frac{n-2}{2}|\nabla f|^{2}\right)\right. \\
& \left.-\frac{\nabla_{i} R}{2(n-1)}+\frac{n-2}{2} \nabla_{i}|\nabla f|^{2}\right] .
\end{aligned}
$$

Since both $|\nabla f|$ and $|\nabla \nabla f|$ are bounded on $\left[0, T_{\infty}\right)$ we may use Theorem 4.2 in [10] again to get $\|\nabla f\|_{C^{\alpha}\left(M \times\left[0, T_{\infty}\right)\right)} \leq C$. Hence we obtain $\|W\|_{C^{2, \alpha}\left(M \times\left[0, T_{\infty}\right)\right)} \leq C$ from (2.6). To get higher derivatives estimates, we rewrite (1.2) as

$$
f_{t}=H(\bar{R}) e^{-2 f}\left(\frac{R}{2(n-1)}-\Delta f-\frac{n-2}{2}|\nabla f|^{2}\right)+G(0)
$$

where

$$
H(\bar{R})=\frac{G\left(\frac{\bar{R}}{2(n-1)}\right)-G(0)}{\frac{\bar{R}}{2(n-1)}} \text { is a smooth function. }
$$

Thus by linear theory and bounds on $\|W\|_{C^{2, \alpha}\left(M \times\left[0, T_{\infty}\right)\right)}$ and $\|\nabla f\|_{C^{\alpha}\left(M \times\left[0, T_{\infty}\right)\right)}$, we easily get

$$
\|f\|_{C^{4, \alpha}\left(M \times\left[0, T_{\infty}\right)\right)} \leq C .
$$

Then by (2.6) we have $\|W\|_{C^{5, \alpha}\left(M \times\left[0, T_{\infty}\right)\right)} \leq C$, which implies that

$$
\|f\|_{C^{7, \alpha}\left(M \times\left[0, T_{\infty}\right)\right)} \leq C
$$

from (2.7). Hence we can bootstrap to get all the $C^{k, \alpha}$ norms of $f$ bounded on $\left[0, T_{\infty}\right)$, and then we can extend the solution $f$ to a larger time interval, which gives us the desired contradiction and the proof of Theorem 2.1 is completed.

Corollary 2.4. If we assume that $(M, g)$ is a connected compact locally conformally flat manifold with scalar curvature $R>0$, then either $T_{\infty}=\infty$ or $\sup _{M \times\left[0, T_{\infty}\right)}|f|=\infty$.

Proof. The Aleksandrov reflection method in Ye [11] can be used to get

$$
|\nabla f| \leq C
$$


for some $C$ depending only on $F$ and $f_{0}$, hence the corollary follows easily.

\section{Expanding flows}

From now on, we shall assume that $F^{\prime}<0$ and $F>0$, which we refer to as the expanding flow because $f$ is always increasing under the flow. Intuitively, since the metric is getting bigger, we expect that the manifold will become flatter after long time. In fact, we can show that the scalar curvature $\bar{R}$ is exponentially decaying.

Lemma 3.1. $|\bar{R}| \leq C e^{-C_{0} t}$ for some positive constants $C$ and $C_{0}$ depending only on $n, F$ and $f_{0}{ }^{2}$

Proof. We first prove that $\bar{R}$ is bounded. Define $\bar{R}_{\max }=\max _{x \in M} \bar{R}(x, t)$ and $\bar{R}_{\min }=\min _{x \in M} \bar{R}(x, t)$. By maximum principle and (2.5), we get

$$
\frac{d}{d t} \bar{R}_{\max } \leq-F\left(\bar{R}_{\max }\right) \bar{R}_{\max } \leq 0 \text { if } \bar{R}_{\max } \geq 0
$$

Similarly,

$$
\frac{d}{d t} \bar{R}_{\min } \geq-F\left(\bar{R}_{\min }\right) \bar{R}_{\min } \geq 0 \text { if } \bar{R}_{\min } \leq 0
$$

From (3.1) and (3.2), there exists some $N$ such that $|\bar{R}| \leq N$, hence $F(\bar{R}) \geq C_{0}>0$. To show that $\bar{R}_{\max } \leq C e^{-C_{0} t}$ we consider the following two cases:

Case I. If $\bar{R}_{\max }>0 \forall t$, we have

$$
\frac{d}{d t} \bar{R}_{\max } \leq-F\left(\bar{R}_{\max }\right) \bar{R}_{\max } \leq-C_{0} \bar{R}_{\max }
$$

therefore $\bar{R}_{\max }(t) \leq \bar{R}_{\max }(0) e^{-C_{0} t}$.

Case II . If $\bar{R}_{\max }$ first becomes nonpositive at $t_{0} \in\left[0, T_{\infty}\right)$, then by maximum principle we have $\bar{R}_{\max }(t) \leq 0 \forall t \in\left[t_{0}, T_{\infty}\right)$. Therefore in both

\footnotetext{
${ }^{2}$ From now on, unless otherwise stated, all the constants are positive uniform constants depending only on $n, F$ and initial condition.
} 
cases we have $\bar{R}_{\max } \leq C e^{-C_{0} t}$ for all $t . \bar{R}_{\min } \geq-C e^{-C_{0} t}$ can be proved analogously.

Corollary 3.2. $\frac{1}{C} t-C^{\prime} \leq f \leq C t+C^{\prime}$ for some constants $C$ and $C^{\prime}$.

Proposition 3.3. There exists some constant $C$ depending only on $n, f_{0}$ and $F$ such that

$$
|\nabla f| \leq C
$$

Proof. Recall that

$$
\begin{aligned}
& \partial_{t}|\nabla f|^{2}=-G^{\prime} e^{-2 f}\left[\Delta|\nabla f|^{2}-2|\nabla \nabla f|^{2}-2 R_{i l} \nabla_{i} f \nabla_{l} f\right. \\
& \left.+4|\nabla f|^{2}\left(\frac{R}{2(n-1)}-\Delta f-\frac{(n-2)}{2}|\nabla f|^{2}\right)-\frac{\nabla f \nabla R}{(n-1)}+(n-2) \nabla f \nabla|\nabla f|^{2}\right] .
\end{aligned}
$$

Using $\bar{R}=2(n-1) e^{-2 f}\left(\frac{R}{2(n-1)}-\Delta f-\frac{n-2}{2}|\nabla f|^{2}\right)$ and applying maximum principle to $\Psi=\max _{x \in M}|\nabla f|^{2}$ we can get the following inequality

$$
\frac{d}{d t} \Psi \leq\left(C e^{-2 f}+C|\bar{R}|\right) \Psi+C e^{-2 f} .
$$

Hence by Lemma 3.1 and Corollary 3.2 it follows easily that $\Psi \leq C$.

We should comment that the gradient estimate for this problem is easy, but in the cases of Ricci and Yamabe flow the gradient estimates are hard and the key. ${ }^{3}$

By Theorem 2.1, Corollary 3.2 and Proposition 3.3 we have

Proposition 3.4. For expanding flow, the solution $f$ to (1.2) exists for all time i.e., $T_{\infty}=\infty$.

\section{Uniform estimates for $|\Delta f|$ and $|\nabla \nabla f|$.}

The Hessian estimate is central when studying general nonlinear parabolic equations. In fact, with the extra assumption that $F$ is concave, a uniform $C^{2}$ estimate combined with Krylov's theorem (see [9]) yield

\footnotetext{
${ }^{3}$ We would like to thank the referee for pointing out this to us.
} 
a uniform $C^{2, \alpha}$ estimate of the solution. We then can bootstrap to get all the higher derivatives estimates. Although Krylov's theorem does not apply to more general case without assuming concavity of $F$, a uniform Hessian estimate is still the first essential step for getting higher derivatives estimates.

In this section we are going to prove the Hessian estimate.

Proposition 4.1. There exists a constant $C$ depending only on $n, F$ and $f_{0}$ such that

$$
|\nabla \nabla f| \leq C .
$$

The strategy of obtaining Hessian estimate is the following :

I. We first obtain an estimate for $\Delta f$. In fact, from (1.3) and Lemma 3.1, we can get a Laplacian estimate depending on time. But to get a uniform estimate for $\Delta f$ we need to calculate the evolution equation for $\Delta f$ then apply maximum principle.

II. We then use the estimate for $\Delta f$ to prove an estimate for $|\nabla \nabla f|$ by estimating a quantity involving both $\Delta f$ and $|\nabla \nabla f|$. The reason for doing so will become clear as we proceed in our proof.

We shall start with the estimate of $\Delta f$.

Lemma 4.2. There exists a constant $C$ depending only on $n, F$ and $f_{0}$ such that

$$
|\Delta f| \leq C .
$$

Proof. By straightforward computation we have

$$
\begin{aligned}
& (\Delta f)_{t}=-e^{-2 f} G^{\prime}\left[\Delta \Delta f-\frac{\Delta R}{2(n-1)}+\frac{n-2}{2} \Delta|\nabla f|^{2}\right. \\
& +\left(2 \Delta f-4|\nabla f|^{2}\right)\left(\frac{R}{2(n-1)}-\Delta f-\frac{n-2}{2}|\nabla f|^{2}\right) \\
& \left.+4 \nabla f\left(\frac{\nabla R}{2(n-1)}-\nabla \Delta f-\frac{n-2}{2} \nabla|\nabla f|^{2}\right)\right] \\
& +e^{-4 f} G^{\prime \prime}\left[4|\nabla f|^{2}\left(\frac{R}{2(n-1)}-\Delta f-\frac{n-2}{2}|\nabla f|^{2}\right)^{2}+\frac{|\nabla R|^{2}}{4(n-1)^{2}}+|\nabla \Delta f|^{2}\right. \\
& +\left.\left.\frac{(n-2)^{2}}{4}|\nabla| \nabla f\right|^{2}\right|^{2}-\frac{\nabla \Delta f \nabla R}{n-1}+(n-2) \nabla \Delta f \nabla|\nabla f|^{2}-\frac{n-2}{2(n-1)} \nabla R \nabla|\nabla f|^{2} \\
& \left.+4 \nabla f\left(\frac{-\nabla R}{2(n-1)}+\nabla \Delta f+\frac{n-2}{2} \nabla|\nabla f|^{2}\right)\left(\frac{R}{2(n-1)}-\Delta f-\frac{n-2}{2}|\nabla f|^{2}\right)\right] .
\end{aligned}
$$


It is readily seen that the main bad terms in the evolution of $\Delta f$ are terms involving $|\nabla \nabla f|$ and $|\nabla \nabla f|^{2}$. To control these bad terms, we shall make use of the negative $|\nabla \nabla f|^{2}$ in the evolution equation of $|\nabla f|^{2}$ and consider the quantity $\varphi=\Delta f+\lambda|\nabla f|^{2}$, where $\lambda$ is a number to be determined later. We have

$$
\begin{aligned}
& \varphi_{t}=-G^{\prime} e^{-2 f}\left[\Delta \varphi-\frac{\Delta R}{2(n-1)}+(n-2-2 \lambda) R_{i l} \nabla_{i} f \nabla_{l} f+(n-6) \nabla f \nabla \varphi\right. \\
& +(n-2-2 \lambda)|\nabla \nabla f|^{2}+(4 \lambda-2 n+4) \nabla f \nabla|\nabla f|^{2} \\
& \left.+\frac{2-\lambda}{n-1} \nabla f \nabla R+\left(2 \varphi+(2 \lambda-4)|\nabla f|^{2}\right)\left(\frac{R}{2(n-1)}-\Delta f-\frac{n-2}{2}|\nabla f|^{2}\right)\right] \\
& +e^{-4 f} G^{\prime \prime}\left[4|\nabla f|^{2}\left(\frac{R}{2(n-1)}-\Delta f-\frac{n-2}{2}|\nabla f|^{2}\right)^{2}+\frac{|\nabla R|^{2}}{4(n-1)^{2}}+\nabla \Delta f \nabla \varphi-\frac{\nabla R \nabla \varphi}{n-1}\right. \\
& +(n-2-\lambda) \nabla \varphi \nabla|\nabla f|^{2}+\left.\left.\left(\lambda^{2}+\frac{(n-2)^{2}}{4}-(n-2) \lambda\right)|\nabla| \nabla f\right|^{2}\right|^{2} \\
& +\left(\frac{\lambda}{n-1}-\frac{n-2}{2(n-1)}\right) \nabla R \nabla|\nabla f|^{2}+4 \nabla f\left(\frac{-\nabla R}{2(n-1)}+\nabla \varphi-\lambda \nabla|\nabla f|^{2}+\frac{n-2}{2} \nabla|\nabla f|^{2}\right) \\
& \left.+\left(\frac{R}{2(n-1)}-\Delta f-\frac{n-2}{2}|\nabla f|^{2}\right)\right] .
\end{aligned}
$$

Here we used

$$
\nabla \Delta f=\nabla \varphi-\lambda \nabla|\nabla f|^{2}
$$

and

$$
|\nabla \Delta f|^{2}=\nabla \Delta f \nabla \varphi-\lambda \nabla \varphi \nabla|\nabla f|^{2}+\left.\left.\lambda^{2}|\nabla| \nabla f\right|^{2}\right|^{2}
$$

Define $\varphi_{\max }=\max _{x \in M} \varphi(x, t)$. Substituting

$$
\frac{R}{2(n-1)}-\Delta f-\frac{n-2}{2}|\nabla f|^{2}=\frac{e^{2 f} \bar{R}}{2(n-1)}
$$

and using the estimate on $|\nabla f|$ we have

$$
\begin{aligned}
& \frac{d}{d t} \varphi_{\max } \leq-G^{\prime} e^{-2 f}\left[(n-2-2 \lambda)|\nabla \nabla f|^{2}+C_{10}(-2 n+4+4 \lambda)|\nabla \nabla f|\right. \\
& \left.+C_{11} \lambda+C_{12}+\frac{e^{2 f}|\bar{R}|}{2(n-1)}\left(2\left|\varphi_{\max }\right|+C_{13} \lambda+C_{14}\right)\right] \\
& +\left|G^{\prime \prime}\right| e^{-4 f}\left[C_{15} e^{4 f} \bar{R}^{2}+\left(C_{16} \lambda^{2}+C_{17}\right)|\nabla \nabla f|^{2}\right. \\
& \left.+\left(\left(C_{18}+C_{19} \lambda\right)|\nabla \nabla f|+C_{20}\right) e^{2 f}|\bar{R}|+\left(C_{21}+C_{22} \lambda\right)|\nabla \nabla f|+C_{23}\right] .
\end{aligned}
$$

Let $\lambda=n-1$ and use Lemma 3.1 and Corollary 3.2, we obtain

$$
\frac{d}{d t} \varphi_{\max } \leq C_{24} \bar{R}^{2}+C_{25}|\bar{R}|+C_{26}|\bar{R}|\left|\varphi_{\max }\right|+C_{27} e^{-2 f} \text { for } t \geq T_{1}
$$


where $T_{1}$ is a positive number. Therefore we can integrate (4.1) to get $\varphi_{\max } \leq C$. In the same manner we can show $\varphi_{\min } \geq-C$ by choosing $\lambda$ negative. Hence we have proved $|\Delta f| \leq C$.

We are going to use the estimate on $\Delta f$ to prove an estimate on $|\nabla \nabla f|$. Let's compute the evolution equation for $|\nabla \nabla f|^{2}$. We have

$$
\left(|\nabla \nabla f|^{2}\right)_{t}=2 \nabla_{i} \nabla_{j} f \nabla_{i} \nabla_{j} f_{t}
$$

Straightforward computation shows that

$$
\begin{aligned}
& \nabla_{i} \nabla_{j} f_{t}=-e^{-2 f} G^{\prime}\left[\nabla_{i} \nabla_{j} \Delta f-\frac{\nabla_{i} \nabla_{j} R}{2(n-1)}+\frac{n-2}{2} \nabla_{i} \nabla_{j}|\nabla f|^{2}\right. \\
& +2 \nabla_{i} \nabla_{j} f\left(\frac{R}{2(n-1)}-\Delta f-\frac{n-2}{2}|\nabla f|^{2}\right)+2 \nabla_{j} f\left(\frac{\nabla_{i} R}{2(n-1)}-\nabla_{i} \Delta f-\frac{n-2}{2} \nabla_{i}|\nabla f|^{2}\right) \\
& \left.+2 \nabla_{i} f\left(\frac{\nabla_{j} R}{2(n-1)}-\nabla_{j} \Delta f-\frac{n-2}{2} \nabla_{j}|\nabla f|^{2}\right)-4 \nabla_{i} f \nabla_{j} f\left(\frac{R}{2(n-1)}-\Delta f-\frac{n-2}{2}|\nabla f|^{2}\right)\right] \\
& +e^{-4 f} G^{\prime \prime}\left[2 \nabla_{i} f\left(\frac{R}{2(n-1)}-\Delta f-\frac{n-2}{2}|\nabla f|^{2}\right)-\frac{\nabla_{i} R}{2(n-1)}+\nabla_{i} \Delta f+\frac{n-2}{2} \nabla_{i}|\nabla f|^{2}\right] \\
& \cdot\left[2 \nabla_{j} f\left(\frac{R}{2(n-1)}-\Delta f-\frac{n-2}{2}|\nabla f|^{2}\right)-\frac{\nabla_{j} R}{2(n-1)}+\nabla_{j} \Delta f+\frac{n-2}{2} \nabla_{j}|\nabla f|^{2}\right] .
\end{aligned}
$$

Therefore, by all the estimates we have so far, we can get

Lemma 4.3. (a)

$$
\begin{aligned}
& \left(|\nabla \nabla f|^{2}\right)_{t} \leq-e^{-2 f} G^{\prime}\left(\Delta|\nabla \nabla f|^{2}-\frac{3}{2}|\nabla \nabla \nabla f|^{2}\right. \\
& \left.+C_{28}|\nabla \nabla f|^{3}+C_{29}|\nabla \nabla f||\nabla \Delta f|+C_{30}\right) \\
& +e^{-4 f}\left|G^{\prime \prime}\right|\left(C_{31}|\nabla \nabla f|^{3}+C_{32}|\nabla \Delta f|^{2}|\nabla \nabla f|\right. \\
& \left.+C_{33}|\nabla \Delta f||\nabla \nabla f|^{2}+C_{34}\right) .
\end{aligned}
$$

and (b)

$$
\left(|\nabla f|^{2}\right)_{t} \leq-e^{-2 f} G^{\prime}\left(\Delta|\nabla f|^{2}-|\nabla \nabla f|^{2}+C_{35}\right)
$$

From Lemma 4.3(a), it is easy to see that the bad terms in the evolution of $|\nabla \nabla f|^{2}$ are $|\nabla \Delta f||\nabla \nabla f|^{2},|\nabla \Delta f|^{2}|\nabla \nabla f|,|\nabla \nabla f|^{3}$ etc.. Now the idea is to produce good terms from some function of $\Delta f$, then use these good terms to dominate those bad terms. We let $a=\sup _{M \times[0, \infty)}|\Delta f|+1$ and consider the quantity $Q=\Delta f+a$. 
Calculating the evolution for $Q^{2}$ and using estimates on $|\nabla f|$ and $\Delta f$, we have

$$
\begin{aligned}
& \left(Q^{2}\right)_{t} \leq-e^{-2 f} G^{\prime}\left(\Delta Q^{2}-2|\nabla \Delta f|^{2}+C_{36}+C_{37}|\nabla \Delta f|+C_{38}|\nabla \nabla f|^{2}\right) \\
& +e^{-4 f}\left|G^{\prime \prime}\right|\left(C_{39}+C_{40}|\nabla \Delta f|^{2}+C_{41}|\nabla \nabla f|^{2}\right) \\
& \leq-e^{-2 f} G^{\prime}\left(\Delta Q^{2}-|\nabla \Delta f|^{2}+C_{42}+C_{43}|\nabla \nabla f|^{2}\right) \text { for } t \geq T_{2} .
\end{aligned}
$$

If we multiply $\left(Q^{2}\right)_{t}$ by $|\nabla \nabla f|^{2}$ then we get a negative $|\nabla \Delta f|^{2}|\nabla \nabla f|^{2}$ term to dominate those bad terms such as $|\nabla \Delta f||\nabla \nabla f|^{2}$ and $|\nabla \Delta f|^{2}|\nabla \nabla f|$, but at the same time we also get a bad positive $|\nabla \nabla f|^{4}$ term, hence we need to add in some negative $|\nabla \nabla f|^{4}$ term which can be produced by multiplying the evolution of $|\nabla f|^{2}$ by $|\nabla \nabla f|^{2}$. We shall consider the quantity

$$
\omega=\left(Q^{2}+\beta\right)|\nabla \nabla f|^{2}+\gamma|\nabla f|^{2}|\nabla \nabla f|^{2},
$$

where $\beta$ and $\gamma$ are numbers to be chosen later. By computing the evolution equation for $\omega$ and using the estimates on $|\nabla f|$ and $\Delta f$, we have the following inequality

$$
\begin{aligned}
& \omega_{t} \leq-e^{-2 f} G^{\prime}\left[\Delta \omega+C_{44}|\nabla \nabla f||\nabla \Delta f||\nabla \nabla \nabla f|-|\nabla \Delta f|^{2}|\nabla \nabla f|^{2}\right. \\
& +C_{45} \gamma|\nabla \nabla f|^{2}|\nabla \nabla \nabla f|+\left(C_{46}+C_{47} \gamma\right)|\nabla \nabla f|^{2}+\left(C_{48}-\gamma\right)|\nabla \nabla f|^{4} \\
& -\frac{3}{2} \beta|\nabla \nabla \nabla f|^{2}+\left(\beta+\gamma+C_{49}\right)\left(C_{50}|\nabla \nabla f|^{3}\right. \\
& \left.\left.+C_{51}|\nabla \nabla f||\nabla \Delta f|+C_{52}\right)\right] \\
& +e^{-4 f}\left|G^{\prime \prime}\right|\left(\beta+\gamma+C_{53}\right)\left(C_{54}|\nabla \nabla f|^{3}+C_{55}|\nabla \Delta f||\nabla \nabla f|^{2}\right. \\
& \left.+C_{56}|\nabla \Delta f|^{2}|\nabla \nabla f|+C_{57}\right) \text { for } t \geq T_{3} .
\end{aligned}
$$

Let $\gamma=C_{48}+2$ and choose $\beta$ so large that

$$
\frac{\beta}{2}|\nabla \nabla \nabla f|^{2}+\frac{1}{2}|\nabla \Delta f|^{2}|\nabla \nabla f|^{2} \geq C_{44}|\nabla \nabla f||\nabla \Delta f||\nabla \nabla \nabla f|
$$

and

$$
\beta|\nabla \nabla \nabla f|^{2}+|\nabla \nabla f|^{4} \geq C_{45} \gamma|\nabla \nabla f|^{2}|\nabla \nabla \nabla f| .
$$

Then the inequality (4.2) becomes

$$
\begin{aligned}
& \omega_{t} \leq-e^{-2 f} G^{\prime}\left(\Delta \omega-\frac{1}{2}|\nabla \Delta f|^{2}|\nabla \nabla f|^{2}-|\nabla \nabla f|^{4}\right. \\
& +C_{58}+C_{59}|\nabla \nabla f|^{3}+C_{60}|\nabla \Delta f||\nabla \nabla f| \\
& \left.+C_{61}|\nabla \Delta f|^{2}|\nabla \nabla f|+C_{62}|\nabla \Delta f||\nabla \nabla f|^{2}\right) \text { for } t \geq T_{4} .
\end{aligned}
$$


To take care of the bad terms in (4.3) we have

$$
\begin{aligned}
& -\frac{1}{2}|\nabla \Delta f|^{2}|\nabla \nabla f|^{2}+C_{61}|\nabla \Delta f|^{2}|\nabla \nabla f|+C_{62}|\nabla \Delta f||\nabla \nabla f|^{2}+C_{60}|\nabla \Delta f||\nabla \nabla f| \\
& =\left(-\frac{1}{6}|\nabla \Delta f|^{2}+C_{62}|\nabla \Delta f|\right)|\nabla \nabla f|^{2}+\left(-\frac{1}{6}|\nabla \nabla f|^{2}+C_{61}|\nabla \nabla f|\right)|\nabla \Delta f|^{2} \\
& +\left(-\frac{1}{6}|\nabla \Delta f|^{2}|\nabla \nabla f|^{2}+C_{60}|\nabla \Delta f||\nabla \nabla f|\right) \\
& \leq C_{63}|\nabla \nabla f|^{2}+C_{64} \text { if }|\nabla \nabla f| \geq 6 C_{61} .
\end{aligned}
$$

For the other terms, we have

$$
-|\nabla \nabla f|^{4}+C_{59}|\nabla \nabla f|^{3} \leq-\frac{1}{2}|\nabla \nabla f|^{4}+C_{65}
$$

Therefore we obtain the following inequality by maximum principle

$$
\frac{d}{d t} \omega_{\max } \leq 0 \text { if } \omega_{\max } \geq C_{66} \text { and } t \geq T_{4},
$$

where $\omega_{\max }=\max _{x \in M} \omega(x, t)$. Hence we have shown $\omega=\left(Q^{2}+\beta\right)|\nabla \nabla f|^{2}+$ $\gamma|\nabla f|^{2}|\nabla \nabla f|^{2} \leq C$ and the proof of proposition 4.1 is completed.

Since $|\nabla \nabla f|$ is bounded, the inequality Lemma 4.3(a) now becomes

$$
\begin{aligned}
& \left(|\nabla \nabla f|^{2}\right)_{t} \leq-e^{-2 f} G^{\prime}\left(\Delta|\nabla \nabla f|^{2}-\frac{3}{2}|\nabla \nabla \nabla f|^{2}\right. \\
& \left.+C_{67}|\nabla \Delta f|+C_{68}\right) \\
& +e^{-4 f}\left|G^{\prime \prime}\right|\left(C_{69}|\nabla \Delta f|^{2}+C_{70}|\nabla \Delta f|+C_{71}\right) \\
& \leq-e^{-2 f} G^{\prime}\left(\Delta|\nabla \nabla f|^{2}-|\nabla \nabla \nabla f|^{2}+C_{72}\right) \text { for } t \geq T_{5} .
\end{aligned}
$$

Here we used $|\nabla \nabla \nabla f|^{2} \geq \frac{1}{n}|\nabla \Delta f|^{2}$.

\section{Uniform $|\nabla \Delta f|$ estimate .}

In general, for fully nonlinear parabolic equations, a uniform Hessian estimate is not strong enough for bootstrapping to obtain all the higher derivatives estimates. Therefore we still need to estimate some other higher derivatives of $f$. We shall see in section 7 that a uniform estimate on $|\Delta \Delta f|$ 
is good enough for bootstrapping to get all the $C^{k, \alpha}$ estimates. In this section we are going to get an uniform estimate on $|\nabla \Delta f|$, and in the next section we will use this estimate to prove an uniform estimate on $|\Delta \Delta f|$.

Proposition 5.1. There exists a constant $C$ depending only on $n, F$ and $f_{0}$ such that

$$
|\nabla \Delta f| \leq C
$$

Proof. The idea in proving this proposition is similar to that used in section 4. We first compute the evolution equation for $|\nabla \Delta f|^{2}$ to see what kind of bad terms we have in the equation, then we try to make use of the good negative $|\nabla \nabla \nabla f|^{2}$ term in the evolution of $|\nabla \nabla f|^{2}$. It turns out that the quantity $\chi=\left(|\nabla \nabla f|^{2}+\nu\right)|\nabla \Delta f|^{2}$ is the right quantity to look at. With some patience we calculate that

$$
\begin{aligned}
& \left(\nabla_{i} \Delta f\right)_{t}=-e^{-2 f} G^{\prime}\left[\nabla_{i} \Delta \Delta f-\frac{\nabla_{i} \Delta R}{2(n-1)}+\frac{n-2}{2} \nabla_{i} \Delta|\nabla f|^{2}\right. \\
& +\left(2 \nabla_{i} \Delta f-4 \nabla_{i}|\nabla f|^{2}\right)\left(\frac{R}{2(n-1)}-\Delta f-\frac{n-2}{2}|\nabla f|^{2}\right)+\left(2 \Delta f-4|\nabla f|^{2}\right) \\
& \cdot\left(\frac{\nabla_{i} R}{2(n-1)}-\nabla_{i} \Delta f-\frac{n-2}{2} \nabla_{i}|\nabla f|^{2}\right)+4 \nabla_{i} \nabla_{j} f\left(\frac{\nabla_{j} R}{2(n-1)}-\nabla_{j} \Delta f-\frac{n-2}{2} \nabla_{j}|\nabla f|^{2}\right) \\
& \left.+4 \nabla_{j} f\left(\frac{\nabla_{i} \nabla_{j} R}{2(n-1)}-\nabla_{i} \nabla_{j} \Delta f-\frac{n-2}{2} \nabla_{i} \nabla_{j}|\nabla f|^{2}\right)-2 A \nabla_{i} f\right] \\
& +G^{\prime \prime} e^{-4 f}\left\{4 \nabla_{i}|\nabla f|^{2}\left(\frac{R}{2(n-1)}-\Delta f-\frac{n-2}{2}|\nabla f|^{2}\right)^{2}+8|\nabla f|^{2}\left(\frac{R}{2(n-1)}-\Delta f-\frac{n-2}{2}|\nabla f|^{2}\right)\right. \\
& \cdot\left(\frac{\nabla_{i} R}{2(n-1)}-\nabla_{i} \Delta f-\frac{n-2}{2} \nabla_{i}|\nabla f|^{2}\right)+\frac{\nabla_{i}|\nabla R|^{2}}{4(n-1)^{2}}+2 \nabla_{i} \nabla_{j} \Delta f \nabla_{j} \Delta f+\left.\left.\frac{(n-2)^{2}}{4} \nabla_{i}|\nabla| \nabla f\right|^{2}\right|^{2} \\
& -\frac{1}{(n-1)}\left(\nabla_{i} \nabla_{j} \Delta f \nabla_{j} R+\nabla_{j} \Delta f \nabla_{i} \nabla_{j} R\right)+(n-2)\left(\nabla_{i} \nabla_{j} \Delta f \nabla_{j}|\nabla f|^{2}+\nabla_{j} \Delta f \nabla_{i} \nabla_{j}|\nabla f|^{2},\right. \\
& -\frac{(n-2)}{2(n-1)}\left(\nabla_{i} \nabla_{j} R \nabla_{j}|\nabla f|^{2}+\nabla_{j} R \nabla_{i} \nabla_{j}|\nabla f|^{2}\right)+4 \nabla_{i} \nabla_{j} f\left(\frac{-\nabla_{j} R}{2(n-1)}+\nabla_{j} \Delta f+\frac{n-2}{2} \nabla_{j}|\nabla f|^{2}\right. \\
& \cdot\left(\frac{R}{2(n-1)}-\Delta f-\frac{n-2}{2}|\nabla f|^{2}\right)+4 \nabla_{j} f\left(\frac{-\nabla_{i} \nabla_{j} R}{2(n-1)}+\nabla_{i} \nabla_{j} \Delta f+\frac{n-2}{2} \nabla_{i} \nabla_{j}|\nabla f|^{2}\right) \\
& \cdot\left(\frac{R}{2(n-1)}-\Delta f-\frac{n-2}{2}|\nabla f|^{2}\right)+4 \nabla_{j} f\left(\frac{-\nabla_{j} R}{2(n-1)}+\nabla_{j} \Delta f+\frac{n-2}{2} \nabla_{j}|\nabla f|^{2}\right) \\
& \cdot\left(\frac{\nabla_{i} R}{2(n-1)}-\nabla_{i} \Delta f-\frac{n-2}{2} \nabla_{i}|\nabla f|^{2}\right)-4 B \nabla_{i} f+A\left[2 \nabla_{i} f\left(\frac{R}{2(n-1)}-\Delta f-\frac{n-2}{2}|\nabla f|^{2}\right)\right. \\
& \left.\left.-\frac{\nabla_{i} R}{2(n-1)}+\nabla_{i} \Delta f+\frac{n-2}{2} \nabla_{i}|\nabla f|^{2}\right\}\right\} \\
& -B G^{\prime \prime \prime} e^{-6 f}\left[2 \nabla_{i} f\left(\frac{R}{2(n-1)}-\Delta f-\frac{n-2}{2}|\nabla f|^{2}\right)-\frac{\nabla_{i} R}{2(n-1)}+\nabla_{i} \Delta f+\frac{n-2}{2} \nabla_{i}|\nabla f|^{2}\right]
\end{aligned}
$$


where

$$
\begin{aligned}
& A=\Delta \Delta f-\frac{\Delta R}{2(n-1)}+\frac{n-2}{2} \Delta|\nabla f|^{2}+\left(2 \Delta f-4|\nabla f|^{2}\right)\left(\frac{R}{2(n-1)}-\Delta f-\frac{n-2}{2}|\nabla f|^{2}\right) \\
& +4 \nabla f\left(\frac{\nabla R}{2(n-1)}-\nabla \Delta f-\frac{n-2}{2} \nabla|\nabla f|^{2}\right) \text { and } \\
& B=4|\nabla f|^{2}\left(\frac{R}{2(n-1)}-\Delta f-\frac{n-2}{2}|\nabla f|^{2}\right)^{2}+\frac{|\nabla R|^{2}}{4(n-1)^{2}}+|\nabla \Delta f|^{2}+\left.\left.\frac{(n-2)^{2}}{4}|\nabla| \nabla f\right|^{2}\right|^{2} \\
& -\frac{\nabla R \nabla \Delta f}{n-1}+(n-2) \nabla \Delta f \nabla|\nabla f|^{2}-\frac{n-2}{2(n-1)} \nabla R \nabla|\nabla f|^{2} \\
& +4 \nabla f\left(\frac{-\nabla R}{2(n-1)}+\nabla \Delta f+\frac{n-2}{2} \nabla|\nabla f|^{2}\right)\left(\frac{R}{2(n-1)}-\Delta f-\frac{n-2}{2}|\nabla f|^{2}\right) .
\end{aligned}
$$

By all the estimates we have gotten and the fact that $|\nabla \nabla \Delta f| \geq \frac{1}{\sqrt{n}}|\Delta \Delta f|$ , it is easy to get

$$
\begin{aligned}
& \left(|\nabla \Delta f|^{2}\right)_{t} \leq-e^{-2 f} G^{\prime}\left(\Delta|\nabla \Delta f|^{2}-\frac{3}{2}|\nabla \nabla \Delta f|^{2}+C_{73}\right. \\
& \left.+C_{74}|\nabla \Delta f|^{2}+C_{75}|\nabla \nabla \nabla f||\nabla \Delta f|\right) \\
& +e^{-4 f}\left|G^{\prime \prime}\right|\left(C_{76}+C_{77}|\nabla \Delta f|^{3}+C_{78}|\nabla \nabla \Delta f||\nabla \Delta f|\right. \\
& +C_{79}|\nabla \nabla \Delta f||\nabla \Delta f|^{2}+C_{80}|\nabla \nabla \nabla f||\nabla \Delta f| \\
& \left.+C_{81}|\nabla \nabla \nabla f||\nabla \Delta f|^{2}\right) \\
& +e^{-6 f}\left|G^{\prime \prime \prime}\right|\left(C_{82}+C_{83}|\nabla \Delta f|^{4}\right) \text { for } t \geq T_{6} .
\end{aligned}
$$

We then consider the evolution equation for the quantity $\chi=$ $|\nabla \Delta f|^{2}\left(|\nabla \nabla f|^{2}+\nu\right)$, where $\nu$ is a number to be determined later. From (4.5) and (5.1) we have

$$
\begin{aligned}
& \chi_{t} \leq-e^{-2 f} G^{\prime}\left[\Delta \chi+C_{84}|\nabla \nabla \Delta f||\nabla \nabla \nabla f||\nabla \Delta f|\right. \\
& -\frac{3}{2}\left(|\nabla \nabla f|^{2}+\nu\right)|\nabla \nabla \Delta f|^{2}+\left(|\nabla \nabla f|^{2}+\nu\right)\left(C_{73}+C_{74}|\nabla \Delta f|^{2}\right. \\
& \left.\left.+C_{75}|\nabla \nabla \nabla f||\nabla \Delta f|\right)+C_{72}|\nabla \Delta f|^{2}-|\nabla \nabla \nabla f|^{2}|\nabla \Delta f|^{2}\right] \\
& +e^{-4 f}\left|G^{\prime \prime}\right|\left(|\nabla \nabla f|^{2}+\nu\right)\left(C_{76}+C_{77}|\nabla \Delta f|^{3}+C_{78}|\nabla \nabla \Delta f||\nabla \Delta f|\right. \\
& \left.+C_{79}|\nabla \nabla \Delta f||\nabla \Delta f|^{2}+C_{80}|\nabla \nabla \nabla f||\nabla \Delta f|+C_{81}|\nabla \nabla \nabla f||\nabla \Delta f|^{2}\right) \\
& +e^{-6 f}\left|G^{\prime \prime \prime}\right|\left(|\nabla \nabla f|^{2}+\nu\right)\left(C_{82}+C_{83}|\nabla \Delta f|^{4}\right), \text { for } t \geq T_{7} .
\end{aligned}
$$

We let $\nu=2\left(C_{84}+1\right)^{2}$ then

$$
\nu|\nabla \nabla \Delta f|^{2}+\frac{1}{2}|\nabla \nabla \nabla f|^{2}|\nabla \Delta f|^{2} \geq\left(C_{84}+1\right)|\nabla \nabla \Delta f||\nabla \nabla \nabla f||\nabla \Delta f|,
$$


so we have

$$
\begin{aligned}
& \chi_{t} \leq-e^{-2 f} G^{\prime}\left(\Delta \chi-|\nabla \nabla \Delta f||\nabla \nabla \nabla f||\nabla \Delta f|+C_{85}\right. \\
& \left.+C_{86}|\nabla \Delta f|^{2}+C_{87}|\nabla \nabla \nabla f||\nabla \Delta f|-\frac{1}{2}|\nabla \nabla \nabla f|^{2}|\nabla \Delta f|^{2}\right) \\
& +e^{-4 f}\left|G^{\prime \prime}\right|\left(C_{88}+C_{89}|\nabla \Delta f|^{3}+C_{90}|\nabla \nabla \Delta f||\nabla \Delta f|\right. \\
& +C_{91}|\nabla \nabla \Delta f||\nabla \Delta f|^{2}+C_{92}|\nabla \nabla \nabla f||\nabla \Delta f| \\
& \left.+C_{93}|\nabla \nabla \nabla f||\nabla \Delta f|^{2}\right) \\
& +e^{-6 f}\left|G^{\prime \prime \prime}\right|\left(C_{94}+C_{95}|\nabla \Delta f|^{4}\right), \text { for } t \geq T_{7} .
\end{aligned}
$$

Using the fact that $|\nabla \nabla \nabla f| \geq \frac{1}{\sqrt{n}}|\nabla \Delta f|$ we get

$$
-|\nabla \nabla \Delta f||\nabla \nabla \nabla f||\nabla \Delta f| \leq \frac{-1}{\sqrt{n}}|\nabla \nabla \Delta f||\nabla \Delta f|^{2} .
$$

Therefore

$$
\begin{aligned}
& I \equiv-e^{-2 f} G^{\prime}(-|\nabla \nabla \Delta f||\nabla \nabla \nabla f||\nabla \Delta f|) \\
& +e^{-4 f}\left|G^{\prime \prime}\right|\left(C_{90}|\nabla \nabla \Delta f||\nabla \Delta f|+C_{91}|\nabla \nabla \Delta f||\nabla \Delta f|^{2}\right) \\
& \leq-e^{-2 f} G^{\prime}\left(\frac{-1}{2 \sqrt{n}}|\nabla \nabla \Delta f||\nabla \Delta f|^{2}+C_{90}|\nabla \nabla \Delta f||\nabla \Delta f|\right) \text { for } t \geq T_{8} .
\end{aligned}
$$

Hence we have

$$
I \leq 0 \text { if }|\nabla \Delta f| \geq 2 \sqrt{n} C_{90} \text { and } t \geq T_{8} .
$$

For the rest terms in (5.2) we have

$$
\begin{aligned}
& -e^{-2 f} G^{\prime}\left(-\frac{1}{2}|\nabla \nabla \nabla f|^{2}|\nabla \Delta f|^{2}+C_{85}+C_{86}|\nabla \Delta f|^{2}+C_{87}|\nabla \nabla \nabla f||\nabla \Delta f|\right) \\
& +e^{-4 f}\left|G^{\prime \prime}\right|\left(C_{88}+C_{89}|\nabla \Delta f|^{3}+C_{92}|\nabla \nabla \nabla f||\nabla \Delta f|+C_{93}|\nabla \nabla \nabla f||\nabla \Delta f|^{2}\right) \\
& +e^{-6 f}\left|G^{\prime \prime \prime}\right|\left(C_{94}+C_{95}|\nabla \Delta f|^{4}\right) \\
& \leq-e^{-2 f} G^{\prime}\left[\left(-\frac{1}{8}|\nabla \nabla \nabla f|^{2}+C_{96}|\nabla \nabla \nabla f|\right)|\nabla \Delta f|^{2}\right. \\
& +\left(-\frac{1}{8}|\nabla \Delta f|^{2}|\nabla \nabla \nabla f|^{2}+C_{97}|\nabla \Delta f||\nabla \nabla \nabla f|\right) \\
& \left.+\left(-\frac{1}{4 n}|\nabla \Delta f|^{4}+C_{98} e^{-2 f}|\nabla \Delta f|^{3}+C_{99} e^{-4 f}|\nabla \Delta f|^{4}+C_{100}|\nabla \Delta f|^{2}+C_{101}\right)\right] \\
& \leq 0 \text { for } t \geq T_{9} \text { and }|\nabla \Delta f| \geq C_{102} .
\end{aligned}
$$

Here we used $|\nabla \nabla \nabla f|^{2}|\nabla \Delta f|^{2} \geq \frac{1}{n}|\nabla \Delta f|^{4}$. Thus there exist constants $C_{103}$ and $T_{10}$ such that

$$
\frac{d}{d t} \chi_{\max } \leq 0 \text { if } \chi_{\max } \geq C_{103} \text { and } t \geq T_{10}
$$


which proves that $|\nabla \Delta f|$ is uniformly bounded and the proof of Proposition 5.1 is completed.

\section{Uniform $|\Delta \Delta f|$ estimate.}

In this section, we are going to use all the estimates we have gotten so far to show that $|\Delta \Delta f|$ is uniformly bounded. Then in the next section we shall use a bootstrapping argument to bound all the $C^{k, \alpha}$ norms of $f$.

Proposition 6.1. There exists a constant $C$ depending only on $n, F$ and $f_{0}$ such that

$$
|\Delta \Delta f| \leq C
$$

Proof. We first rewrite (5.1) as

$$
\begin{aligned}
& \left(|\nabla \Delta f|^{2}\right)_{t} \leq-e^{-2 f} G^{\prime}\left(\Delta|\nabla \Delta f|^{2}-|\nabla \nabla \Delta f|^{2}\right. \\
& \left.+C_{104}|\nabla \nabla \nabla f|+C_{105}\right) \text { for } t \geq T_{11} .
\end{aligned}
$$

With some lengthy calculations (we omit the details), we can obtain the following inequality.

$$
\begin{aligned}
& \left((\Delta \Delta f)^{2}\right)_{t} \leq-e^{-2 f} G^{\prime}\left[\Delta(\Delta \Delta f)^{2}-|\nabla \Delta \Delta f|^{2}+C_{106}\right. \\
& \left.+C_{107}|\Delta \Delta f|^{2}+C_{108}|\nabla \nabla \Delta f||\Delta \Delta f|+C_{109}|\nabla \nabla \nabla f|^{2}|\Delta \Delta f|\right] \\
& +e^{-4 f}\left|G^{\prime \prime}\right|\left(C_{110}+C_{111}|\Delta \Delta f|^{3}+C_{112}|\nabla \Delta \Delta f||\Delta \Delta f|\right. \\
& \left.+C_{113}|\nabla \nabla \Delta f|^{2}|\Delta \Delta f|+C_{114}|\nabla \nabla \nabla f|^{2}|\Delta \Delta f|\right) \\
& +e^{-6 f}\left|G^{\prime \prime \prime}\right|\left(C_{115}+C_{116}|\Delta \Delta f|^{2}+C_{117}|\nabla \nabla \Delta f||\Delta \Delta f|\right. \\
& \left.+C_{118}|\nabla \nabla \nabla f||\Delta \Delta f|\right)+C_{119} e^{-8 f}\left|G^{(4)}\right||\Delta \Delta f| .
\end{aligned}
$$

We shall consider the quantity

$$
\xi=|\Delta \Delta f|^{2}\left(|\nabla \Delta f|^{2}+\mu\right)+|\Delta \Delta f|^{2}|\nabla \nabla f|^{2},
$$


where $\mu$ will be chosen later. We use (4.5), (6.1) and (6.2), then

$$
\begin{aligned}
& \xi_{t} \leq-e^{-2 f} G^{\prime}\left[\Delta \xi+C_{120}|\nabla \Delta \Delta f||\Delta \Delta f||\nabla \nabla \Delta f|\right. \\
& +C_{121}|\nabla \Delta \Delta f||\Delta \Delta f||\nabla \nabla \nabla f|-|\nabla \nabla \Delta f|^{2}|\Delta \Delta f|^{2} \\
& +C_{122}|\nabla \nabla \nabla f||\Delta \Delta f|^{2}-\mu|\nabla \Delta \Delta f|^{2}-|\nabla \nabla \nabla f|^{2}|\Delta \Delta f|^{2} \\
& +(1+\mu)\left(C_{123}+C_{124}|\Delta \Delta f|^{2}+C_{125}|\nabla \nabla \Delta f||\Delta \Delta f|\right. \\
& \left.\left.+C_{126}|\nabla \nabla \nabla f|^{2}|\Delta \Delta f|\right)\right] \\
& +e^{-4 f}\left|G^{\prime \prime}\right|(1+\mu)\left(C_{127}+C_{128}|\nabla \Delta \Delta f||\Delta \Delta f|\right. \\
& +C_{129}|\nabla \nabla \Delta f|^{2}|\Delta \Delta f|+C_{130}|\Delta \Delta f|^{3} \\
& \left.+C_{131}|\nabla \nabla \nabla f|^{2}|\Delta \Delta f|\right) \\
& +e^{-6 f}\left|G^{\prime \prime \prime}\right|(1+\mu)\left(C_{132}+C_{133}|\Delta \Delta f|^{2}\right. \\
& \left.+C_{134}|\nabla \nabla \Delta f||\Delta \Delta f|+C_{135}|\nabla \nabla \nabla f||\Delta \Delta f|\right) \\
& +C_{136} e^{-8 f}\left|G^{(4)}\right|(1+\mu)|\Delta \Delta f|, \text { for } t \geq T_{12} .
\end{aligned}
$$

Let $\mu$ be a number so large that

$-\frac{\mu}{2}|\nabla \Delta \Delta f|^{2}-\frac{1}{4}|\nabla \nabla \Delta f|^{2}|\Delta \Delta f|^{2} \leq-\left(C_{120}+1\right)|\nabla \Delta \Delta f||\Delta \Delta f||\nabla \nabla \Delta f|$

and

$-\frac{\mu}{2}|\nabla \Delta \Delta f|^{2}-\frac{1}{4}|\nabla \nabla \nabla f|^{2}|\Delta \Delta f|^{2} \leq-\left(C_{121}+1\right)|\nabla \Delta \Delta f||\Delta \Delta f||\nabla \nabla \nabla f|$.

Using the fact that $|\nabla \nabla \Delta f| \geq \frac{1}{\sqrt{n}}|\Delta \Delta f|$ we have

$$
|\nabla \Delta \Delta f||\Delta \Delta f||\nabla \nabla \Delta f| \geq \frac{1}{\sqrt{n}}|\nabla \Delta \Delta f||\Delta \Delta f|^{2} .
$$


Thus (6.3) becomes

$$
\begin{aligned}
& \xi_{t} \leq-e^{-2 f} G^{\prime}\left[\Delta \xi-\frac{1}{\sqrt{n}}|\nabla \Delta \Delta f||\Delta \Delta f|^{2}\right. \\
& -\frac{3}{4}|\nabla \nabla \Delta f|^{2}|\Delta \Delta f|^{2}-\frac{3}{4}|\nabla \nabla \nabla f|^{2}|\Delta \Delta f|^{2} \\
& +C_{137}|\nabla \nabla \nabla f||\Delta \Delta f|^{2}+C_{138}|\Delta \Delta f|^{2}+C_{139} \\
& \left.+C_{140}|\nabla \nabla \Delta f||\Delta \Delta f|+C_{141}|\nabla \nabla \nabla f|^{2}|\Delta \Delta f|\right] \\
& +e^{-4 f}\left|G^{\prime \prime}\right|\left(C_{142}+C_{143}|\nabla \Delta \Delta f||\Delta \Delta f|\right. \\
& +C_{144}|\nabla \nabla \Delta f|^{2}|\Delta \Delta f|+C_{145}|\Delta \Delta f|^{3} \\
& \left.+C_{146}|\nabla \nabla \nabla f|^{2}|\Delta \Delta f|\right) \\
& +e^{-6 f}\left|G^{\prime \prime \prime}\right|\left(C_{147}+C_{148}|\Delta \Delta f|^{2}+C_{149}|\nabla \nabla \Delta f||\Delta \Delta f|\right. \\
& \left.+C_{150}|\nabla \nabla \nabla f||\Delta \Delta f|\right)+C_{151} e^{-8 f}\left|G^{(4)}\right||\Delta \Delta f|, \text { for } t \geq T_{13} .
\end{aligned}
$$

We can group the terms in (6.4) as follows.

$$
\begin{aligned}
& -e^{-2 f} G^{\prime}\left(-\frac{1}{\sqrt{n}}|\nabla \Delta \Delta f||\Delta \Delta f|^{2}\right) \\
& +C_{143} e^{-4 f}\left|G^{\prime \prime}\right||\nabla \Delta \Delta f||\Delta \Delta f| \leq 0, \text { if }|\Delta \Delta f| \geq C_{152} . \\
& -e^{-2 f} G^{\prime}\left(-\frac{1}{4}|\nabla \nabla \Delta f|^{2}|\Delta \Delta f|^{2}+C_{140}|\nabla \nabla \Delta f||\Delta \Delta f|\right) \\
& +C_{144} e^{-4 f}\left|G^{\prime \prime}\right||\nabla \nabla \Delta f|^{2}|\Delta \Delta f|+C_{149} e^{-6 f}\left|G^{\prime \prime \prime}\right||\nabla \nabla \Delta f||\Delta \Delta f| \\
& \leq C_{153} e^{-2 f} \text { if }|\Delta \Delta f| \geq C_{154} \cdot \\
& -e^{-2 f} G^{\prime}\left(-\frac{1}{4}|\nabla \nabla \nabla f|^{2}|\Delta \Delta f|^{2}+C_{137}|\nabla \nabla \nabla f||\Delta \Delta f|^{2}\right. \\
& \left.+C_{141}|\nabla \nabla \nabla f|^{2}|\Delta \Delta f|\right)+e^{-4 f}\left|G^{\prime \prime}\right|\left(C_{146}|\nabla \nabla \nabla f|^{2}|\Delta \Delta f|\right) \\
& +C_{150} e^{-6 f}\left|G^{\prime \prime \prime}\right||\nabla \nabla \nabla f||\Delta \Delta f| \\
& \leq C_{155} e^{-2 f}|\Delta \Delta f|^{2} \text { if }|\Delta \Delta f| \geq C_{156} . \\
& \frac{1}{4} e^{-2 f} G^{\prime}|\nabla \nabla \Delta f|^{2}|\Delta \Delta f|^{2}+C_{145} e^{-4 f}\left|G^{\prime \prime}\right||\Delta \Delta f|^{3} \\
& \leq \frac{1}{4 n} e^{-2 f} G^{\prime}|\Delta \Delta f|^{4}+C_{145} e^{-4 f}\left|G^{\prime \prime}\right||\Delta \Delta f|^{3} \\
& \leq \frac{1}{8 n} e^{-2 f} G^{\prime}|\Delta \Delta f|^{4} \text { if }|\Delta \Delta f| \geq C_{157} .
\end{aligned}
$$

Therefore there exist constants $C_{158}$ and $T_{14}$ such that

$$
\frac{d}{d t} \xi_{\max } \leq 0 \text { if } \xi_{\max } \geq C_{158} \text { and } t \geq T_{14},
$$

which implies that $|\Delta \Delta f|$ is uniformly bounded. 


\section{Rescaling and convergence.}

Using estimates on $|\Delta f|$ and $|\Delta \Delta f|$, we may apply standard elliptic theory to get

$$
\|\Delta f(\cdot, t)\|_{C^{1, \alpha}(M)} \leq C \text { for some } \alpha \in(0,1) .
$$

Define $\tilde{f}=f-G(0) t$, then

$$
\widetilde{f_{t}}=f_{t}-G(0)=G\left(\frac{\bar{R}}{2(n-1)}\right)-G(0) .
$$

We note that the covariant derivatives of $\tilde{f}$ with respect to the space $x$ is the same as those of $f$. By Lemma 3.1 , we have $\widetilde{f}_{t} \rightarrow 0$ exponentially. Hence $\widetilde{f}$ converges to some function $\widetilde{f}_{\infty}$ as $t \rightarrow \infty$. In particular $\widetilde{f}$ is uniformly bounded. Therefore from (7.1) and elliptic Schauder estimate we can get

$$
\|\tilde{f}(\cdot, t)\|_{C^{3, \alpha}(M)} \leq C .
$$

We are going to prove that all the derivatives of $\tilde{f}$ are also uniformly bounded and then it follows easily that $\tilde{f} \stackrel{C^{\infty}}{\longrightarrow} \tilde{f}_{\infty}$.

Proposition 7.1. There exists uniform constants $C_{k}$ depending on $n, F$ and $f_{0}$ such that

$$
\|\tilde{f}(\cdot, t)\|_{C^{k, \alpha}(M)} \leq C_{k}
$$

Proof. Let $\widetilde{R}=e^{2 G(0) t} \bar{R}$ be the corresponding scalar curvature of the metric $\widetilde{g}=e^{2 f} g$. From (2.1), it is easy to calculate the evolution equation for $\widetilde{R}$

$$
\begin{aligned}
& \partial_{t} \widetilde{R}=(1-n) e^{-2 f}\left(F^{\prime}(\bar{R}) \Delta \widetilde{R}+F^{\prime \prime}(\bar{R}) \nabla \bar{R} \nabla \widetilde{R}+(n-2) F^{\prime}(\bar{R}) \nabla f \nabla \widetilde{R}\right) \\
& -\frac{K(\bar{R}) \bar{R}}{2(n-1)} \widetilde{R}
\end{aligned}
$$

where

$$
K(\bar{R})=\frac{2 G\left(\frac{\bar{R}}{2(n-1)}\right)-2 G(0)}{\frac{\bar{R}}{2(n-1)}} \text { is a smooth function. }
$$


Let $\widehat{R}(x, \tau) \equiv \widetilde{R}(x, \sigma(\tau))$, where $\sigma(\tau)$ satisfies the O.D.E.

$$
\left\{\begin{array}{l}
\sigma^{\prime}(\tau)=\exp (2 G(0) \sigma(\tau)) \\
\sigma(0)=0
\end{array}\right.
$$

Then the evolution equation for $\widehat{R}$ is

$$
\begin{aligned}
& \partial_{\tau} \widehat{R}=(1-n) e^{-2 f}\left(F^{\prime}(\bar{R}) \Delta \widehat{R}+F^{\prime \prime}(\bar{R}) \nabla \bar{R} \nabla \widehat{R}+(n-2) F^{\prime}(\bar{R}) \nabla f \nabla \widehat{R}\right) \\
& -\frac{K(\bar{R})}{2(n-1)} \widehat{R}^{2},
\end{aligned}
$$

which satisfies the uniform parabolicity condition. Now define

$$
\begin{aligned}
& \widehat{a}(x, \tau)=F^{\prime}(\bar{R}) e^{-2 \widetilde{f}}, \widehat{b}_{i}(x, \tau)=F^{\prime \prime}(\bar{R}) e^{-2 \widetilde{f}} \nabla_{i} \bar{R}+(n-2) F^{\prime}(\bar{R}) \nabla_{i} f \\
& \text { and } \widehat{c}(x, \tau)=K(\bar{R}) \hat{R} .
\end{aligned}
$$

From (7.3) and recall that

$$
\begin{aligned}
& \bar{R}=2(n-1) e^{-2 f}\left(\frac{R}{2(n-1)}-\Delta f-\frac{n-2}{2}|\nabla f|^{2}\right) \\
& \widetilde{R}=2(n-1) e^{-2 \widetilde{f}}\left(\frac{R}{2(n-1)}-\Delta \tilde{f}-\frac{n-2}{2}|\nabla \tilde{f}|^{2}\right)
\end{aligned}
$$

we easily get $\|\bar{R}(\cdot, t)\|_{C^{1, \alpha}(M)} \leq C$ and $\|\widetilde{R}(\cdot, t)\|_{C^{1, \alpha}(M)} \leq C$, hence

$$
\|\widehat{a}(\cdot, \tau)\|_{C^{1, \alpha}(M)},\left\|\widehat{b}_{i}(\cdot, \tau)\right\|_{C^{\alpha}(M)},\|\widehat{c}(\cdot, \tau)\|_{C^{1, \alpha}(M)} \leq C .
$$

Thus we can apply Schauder estimate(see [8], p.80) to get

$$
\|\widehat{R}(\cdot, \tau)\|_{C^{2, \alpha}(M)} \leq C .
$$

From (7.4) we have

$$
\Delta \tilde{f}+\frac{n-2}{2}|\nabla \tilde{f}|^{2}-\frac{R}{2(n-1)}+\frac{\widetilde{R}}{2(n-1)} e^{2 \tilde{f}}=0 .
$$

Hence by (7.3) and (7.5) we get

$$
\|\widetilde{f}(\cdot, \tau)\|_{C^{4, \alpha}(M)} \leq C .
$$


Because of (7.5) we have

$$
\|\widehat{a}(\cdot, \tau)\|_{C^{2, \alpha}(M)},\left\|\widehat{b}_{i}(\cdot, \tau)\right\|_{C^{1, \alpha}(M)},\|\widehat{c}(\cdot, \tau)\|_{C^{2, \alpha}(M)} \leq C .
$$

Using Schauder estimate again we get $\|\widehat{R}(\cdot, \tau)\|_{C^{3, \alpha}(M)} \leq C$, which implies

$$
\|\tilde{f}(\cdot, \tau)\|_{C^{5, \alpha}(M)} \leq C .
$$

In this way we may bootstrap to get

$$
\|\widehat{R}(\cdot, \tau)\|_{C^{k, \alpha}(M)} \leq C_{k} \text { and }\|\tilde{f}(\cdot, \tau)\|_{C^{k, \alpha}(M)} \leq C_{k} . \quad \text { Q.E.D. }
$$

Proof. [1.3.] By taking the covariant derivatives of (7.2) and using (1.2), we get

$$
\nabla^{k} \tilde{f}_{t}=e^{-2 f}(\text { sum of terms involving derivatives of } \tilde{f}) \text {. }
$$

Therefore $\nabla^{k} \tilde{f_{t}} \longrightarrow 0$ exponentially and hence $\tilde{f} \stackrel{C^{\infty}}{\longrightarrow} \tilde{f}_{\infty}$, which proves Theorem 1.3.

Acknowledgment. I would like to thank Professor Bennett Chow under whose advice this paper was written. I am especially indebted to him for his patient guidance and constant encouragement through the years. I also would like to thank Mr. Dong-Ho Tsai for many helpful discussions. 


\section{References.}

[1] J. Bartz, M. Struwe and R. Ye, A new approach to the Ricci flow on $S^{2}$, Forschungsinstitut für Mathematik ETH Zürich, February 1993.

[2] B. Chow, The Ricci flow on the 2-Sphere, J. Diff. Geom., 33(1991), 325-334.

[3] B. Chow, The Yamabe flow on locally conformally flat manifolds of positive Ricci curvature, Comm. on Pure and Applied Math., Vol. XLV, 10031014.(1992)

[4] B. Chow, L. P. Liou and D. H. Tsai, On the nonlinear parabolic equation $u_{t}=F(\Delta u+n u)$ on $S^{n}$, preprint.

[5] R. Hamilton, The Ricci flow on surfaces, Contemporary Mathematics 71(1988), 237-262.

[6] R. Hamilton, Three-manifolds with positive Ricci curvature, J. Diff. Geom., 17(1982), 255-306.

[7] R. Hamilton, Four-manifolds with positive curvature operator, J. Diff. Geom., 24(1986), 153-179.

[8] J. Jost, Nonlinear Methods in Riemannian and Kählerian Geometry, Birkhäuser, 1988.

[9] N. V. Krylov, Boundedly inhomogeneous elliptic and parabolic equations in a domain. Izv. Akad. Nauk SSSR, 47 (1983), 75-108.

[10] N. V. Krylov and M. V. Safonov, A certain property of solutions of parabolic equation with measurable coefficients, Izv. Akad. Nauk 40(1980) 161-175; English transl., Math. USSR Izv. 16(1981) 151-164.

[11] R. Ye, Global existence and convergence of Yamabe flow, J. Diff. Geom., 39(1994), 35-50.

RECEIVED JANUARY 12, 1995.

School of Mathematics

UNIVERSITY OF MINNESOTA

MINNEAPOLIS, MN 55455 